\title{
Communication-Aware Scheduling Algorithm Based on Heterogeneous Computing Systems
}

\author{
Youlin Ruan ${ }^{1,2}$, Gan $\mathrm{Liu}^{3}$, Jianjun $\mathrm{Han}^{3}$, and Qinghua $\mathrm{Li}^{3}$ \\ ${ }^{1}$ School of Information Engineering, Wuhan University of Technology, 430070 Wuhan, \\ P.R. China \\ ${ }^{2}$ State Key Laboratory for Novel Software Technology, Nanjing University, 210093 Nanjing, \\ P.R. China \\ ${ }^{3}$ Department of Computer Science and Technology, Huazhong University of Science and \\ Technology, 430074 Wuhan, P.R. China \\ ruanyl@126.com
}

\begin{abstract}
This paper proposes a new scheduling model that integrates the communication awareness into task scheduling. A scheduling algorithm is proposed for the new model, which can produce optimal schedule by serializing the communications edges. Experiment results show the significantly improved accuracy and efficiency of the new model and algorithm.
\end{abstract}

Keywords: communication aware; concurrent communication; serializing.

\section{Introduction}

In order to exploit the heterogeneous computing power, a lot of works have focused on solving the task scheduling problem. This problem has been shown to be NPcomplete $^{[1]}$. There are several proposed algorithms dealing with the task scheduling problem in heterogeneous systems. They could be classified into a variety of categories, such as list-scheduling, clustering, duplication-based algorithms, and guided random search methods. The list-based algorithms provide good quality of schedules and their performance is comparable with other categories at a lower scheduling time. The Iso-Level Heterogeneous Allocation ${ }^{[2]}$, the Heterogeneous Earliest-Finish-Time (HEFT) and Critical-Path-on-a-Processor (CROP) techniques ${ }^{[3]}$ are all examples. However, most of the above-mentioned works have in common that they employ an idealized model of the target parallel system and don't deliberate on the impact of communication contentions. Intuition and experiments demonstrated that this model results in inaccurate and inefficient schedules ${ }^{[4]}$. Therefore, in order to exploit the potential of parallel processing, efficient scheduling algorithms under the consideration of the communication requirements must be developed. So, CATS was proposed to schedule parallel tasks by considering the communication requirements of the applications and the network bandwidth ${ }^{[5]}$. Sinnen investigated the incorporation of contention awareness into task scheduling by edge scheduling, which considered heterogeneous links and routing ${ }^{[6]}$. 
In this paper, we propose a communication-aware scheduling that extended the classic list scheduling. The rest of paper is organized as follows. In Section 2, we introduce some definitions and preliminaries. Section 3 describes the new contention model, and investigates the integration of the awareness for the contention in task scheduling. Experimental results are presented in section 4.

\section{Preliminaries}

In task scheduling, the program to be scheduled is represented by a directed acyclic graph(DAG) $\mathrm{G}=(V, E, w, c)$, where $V$ is the set of tasks and $E$ is the set of edges. The size of task $n_{i}$ is $w\left(n_{\mathrm{i}}\right)$. An edge from $n_{i}$ to task $n_{\mathrm{j}}$ is expressed as $e_{\mathrm{ij}}$ and size of the edge is expressed as $c_{\mathrm{ij}}$. Given task $n_{i}$, the set of parent tasks is denoted as $\operatorname{pred}\left(n_{i}\right)$; that is, $\operatorname{pred}\left(n_{i}\right)=\left\{n_{k} \mid e_{k i} \in E\right\}$, and the set of children tasks is denoted as $\operatorname{succ}\left(n_{\mathrm{i}}\right)$; that is $\operatorname{succ}\left(n_{\mathrm{i}}\right)=\left\{n_{k} \mid e_{i k} \in E\right\}$. A task $n_{\mathrm{i}}$ is called a join task if $\left|\operatorname{pred}\left(n_{i}\right)\right| \geq 2$, an entry task if $\left|\operatorname{pred}\left(n_{i}\right)\right|=0$, and an exit task if $\left|\operatorname{succ}\left(n_{\mathrm{i}}\right)\right|=0$.

To describe a schedule $\mathrm{S}$ of a $\mathrm{DAG} \mathrm{G}=(V, E, w, c)$, the following terms are defined: $\operatorname{est}(n, P)$ and $\operatorname{ect}(n, P)$ represent the earliest start time and completion time of $n_{i}$ on processor $P$ respectively, and $w(n, P)$ the execution time of node $n \in \mathrm{V}$ on processor $P \in \mathbf{P}$. Thus, the node's earliest completion time is given by $\operatorname{ect}(n, P)=e s t(n$, $P)+w(n, P)$. Given an edge $e_{i j}$, est $\left(e_{i j}\right)$ and $\operatorname{ect}\left(e_{i j}\right)$ represent the earliest start time and completion time of edge $e_{i j}$ respectively. Thus, the edge's earliest completion time is given by $\operatorname{ect}\left(e_{i j}\right)=\operatorname{est}\left(e_{i j}\right)+c_{\mathrm{ij}}$ and $\operatorname{est}\left(n_{j}, P\right) \geq \operatorname{ect}\left(e_{i j}\right)$, for $n_{\mathrm{i}}, n_{\mathrm{j}} \in V, e_{i j} \in E$.

\section{The Proposed Algorithm}

To make task scheduling contention aware, the communication edges must be serialized because communication cannot be concurrent. Thus, the awareness for communication is serialize achieved by scheduling edge.

Definition 1 (Contention Model): A target parallel system $M_{\text {contention }}=(\mathbf{P}, w$, comm $)$ consists of a set of possibly heterogeneous processors $\mathbf{P}$ connected by the communication network, where $\operatorname{comm}\left(e_{\mathrm{ij}}, P_{s r c}, P_{d s t}\right)$ denotes the communication cost of edge $e_{\mathrm{ij}}$ from processor $P_{s r c}$ to $P_{d s t}$.

When a communication, represented by the edge $e_{i j}$, is performed between two processors $P_{s r c}$ and $P_{d s t}$. Thus, the earliest completion time of $e_{i j}$ is

$$
\operatorname{ect}\left(e_{i j}\right)= \begin{cases}\operatorname{ect}\left(n_{i}\right) & \text { if } P_{s r c}=P_{d s t} \\ \operatorname{ect}\left(n_{i}\right)+\operatorname{comm}\left(e_{i j}, P_{s r c}, P_{d s t}\right) & \text { otherwise }\end{cases}
$$

where $P_{s r c}=\operatorname{proc}\left(n_{i}\right)$ and $P_{d s t}=\operatorname{proc}\left(n_{j}\right)$. Thus, the edge earliest completion time $e c t\left(e_{i j}\right)$ is now the earliest completion time of $n_{\mathrm{i}}$ plus the communication of $e_{i j}$ from $P_{s r c}$ to $P_{d s t}$, unless the communication is local. For $n_{a}$, we claim that a chain of communications can be found as $X_{a}: e_{1 a} \rightarrow e_{2 a} \rightarrow \ldots \rightarrow e_{k a}$, where $n_{i} \in \operatorname{pred}\left(n_{a}\right)(\mathrm{i}=1 . . \mathrm{k})$, 
such that $\operatorname{est}\left(e_{1 a}\right) \leq e s t\left(e_{2 a}\right) \leq \ldots \leq e s t\left(e_{k a}\right)$,i.e., $\operatorname{ect}\left(n_{1}\right) \leq e c t\left(n_{2}\right) \leq \ldots \leq e c t\left(n_{k}\right)$. We define $\Omega\left(e_{i a}\right)$ as the idle communication time before the communication edge $e_{i a}$, is issued as $\Omega\left(e_{i a}\right)=\operatorname{est}\left(e_{i a}\right)-\operatorname{ect}\left(e_{(i-1) a}\right),(1 \leq \mathrm{i} \leq k)$. Thus, est $\left(n_{a}\right)=\operatorname{ect}\left(n_{1}\right)+\sum_{i=1}^{k}\left\{\operatorname{comr}\left(c_{i a}, P_{s r c}, P_{d s t}\right)+\Omega\left(e_{i a}\right)\right\}$.

Given task $n_{\mathrm{a}}, \operatorname{ect}\left(n_{\mathrm{a}}\right)$ is determined according to its parent tasks and communication edges. If task $n_{\mathrm{a}}$ has a

single parent task $n_{\mathrm{i}}$, then $\operatorname{est}\left(n_{\mathrm{a}}\right)=\operatorname{ect}\left(n_{\mathrm{a}}\right)$ If $n_{\mathrm{a}}$ and $n_{\mathrm{i}}$ exist different processors, the execution of $n_{\mathrm{a}}$ is delayed by $\operatorname{ect}\left(n_{i}\right)+\operatorname{comm}\left(e_{i a}, \operatorname{proc}\left(n_{i}\right), \operatorname{proc}\left(n_{a}\right)\right)$. If $n_{\mathrm{a}}$ and $n_{\mathrm{i}}$ exist same processor, the communication time $c_{\mathrm{ia}}$ is negligible and $n_{\mathrm{a}}$ can start at $\operatorname{ect}\left(n_{\mathrm{i}}\right)$.If task $n_{\mathrm{a}}$ has $k$ parent task $n_{1}, n_{2}, \ldots, n_{\mathrm{k}}$, where $\operatorname{ect}\left(n_{1}\right) \leq \operatorname{ect}\left(n_{2}\right) \leq \operatorname{ect}\left(n_{\mathrm{i}}\right) \leq \ldots \leq \operatorname{ect}\left(n_{\mathrm{k}}\right)$. At first, because of earliest completion of $n_{1}$, the start time of edge $e_{1 \text { a }}$ should be first scheduled to $\operatorname{proc}\left(n_{\mathrm{a}}\right), c t_{1}(s c)$ is denoted as the completion time of scheduling the first communication. However, if the parent task $n_{2}$ cannot complete in advance, which means $c t_{1}(s c)=\operatorname{ect}\left(n_{1}\right)+c_{1 \mathrm{a}} \leq \operatorname{ect}\left(n_{2}\right)$, there is an idle time $\Omega_{1}$ between them. Thus, the edge $e_{2 \mathrm{a}}$ must be delayed by $\operatorname{ect}\left(n_{2}\right)$. Next, the procedure continue to schedules the edge $e_{\mathrm{ia}}(2 \leq \mathrm{i} \leq \mathrm{k})$ that satisfies the following condition: $t_{\mathrm{s}}\left(e_{\mathrm{ia}}\right)=\max \left(c t_{\mathrm{i}-1}(s c), \operatorname{ect}\left(n_{\mathrm{i}}\right)\right)$, where $c t_{i}(s c)=c t_{i-1}(s c)+\Omega_{i-1}+c_{i a}$ and

$$
\Omega_{i}= \begin{cases}0 & \text { if } \operatorname{ect}\left(n_{i+1}\right) \leq c t_{i}(s c) \\ \operatorname{ect}\left(n_{i+1}\right)-c t_{i}(s c) & \text { otherwise }\end{cases}
$$

If $k$ th edge is scheduled, $c t_{\mathrm{k}}(s c)$ and $\operatorname{est}\left(n_{\mathrm{a}}\right)$ can be determined. Therefore, $\operatorname{est}\left(n_{\mathrm{a}}\right)=$ $c t_{\mathrm{k}}(s c)$. We can easily prove that $\operatorname{est}\left(n_{a}\right)$ is minimal.

The best known scheduling heuristic is list scheduling. A common and usually good priority is the node's bottom level $b l$, which is the length of the longest path leaving the node. Recursively defined, it is

$$
b l\left(n_{i}\right)=w\left(n_{i}\right)+\max _{n_{j} \in \operatorname{succ}\left(n_{i}\right)}\left\{c_{i j}+b l\left(n_{j}\right)\right\}
$$

Based on above theory and analysis, we propose an extended list scheduling algorithm that considers the communication awareness by reasonable serializing the communication edges, which is listed as below.

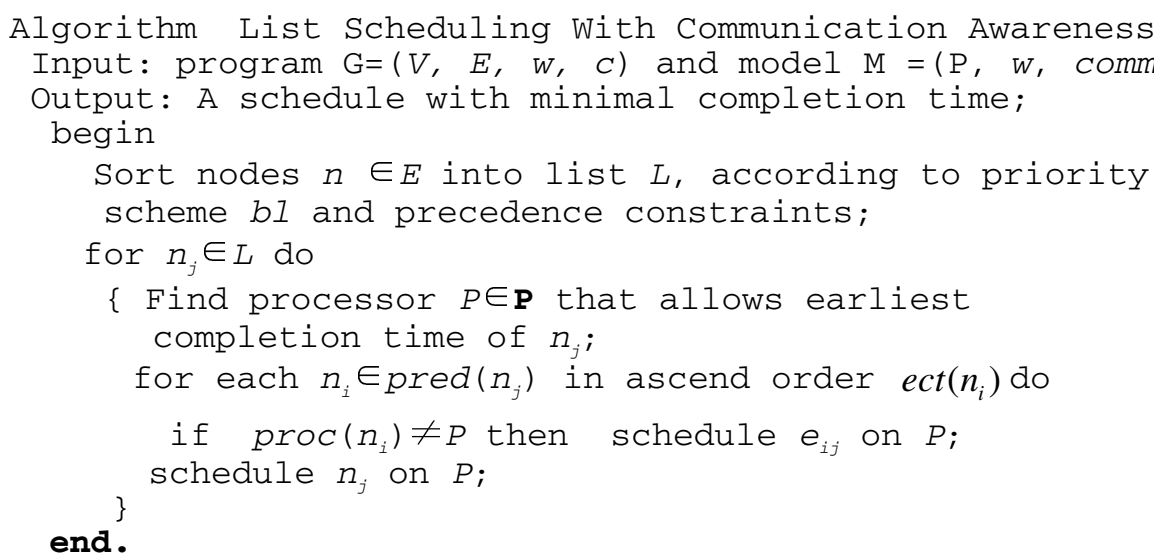




\section{Experiment Results}

This section presents experiments results to verify some aspects of the proposed contention aware scheduling. We adopt the same test methods with [6].In each experiment, the values of these parameters are assigned from the corresponding sets given below.

$S E T_{V}=\{100,200,400,800\}, S E T_{\text {indeg ree }}=\{1,2,5,10\}$ and $S E T_{C C R}=\{0.1,1,5,10\}$.

Fig. 1 and Fig. 2 show the average accuracy and efficiency achieved under the two models over all DAGs on parallel systems. Experimental results demonstrated the significantly improved accuracy and efficiency than classic schedule schemes.

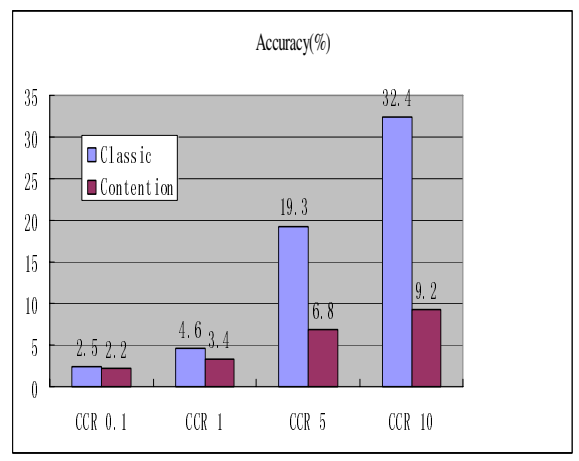

Fig. 1. Average accuracy

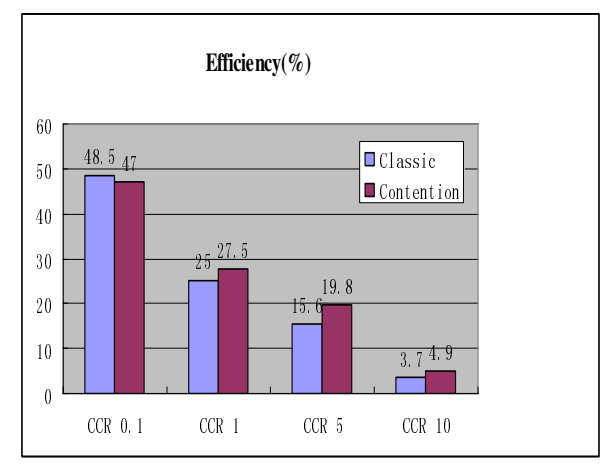

Fig. 2. Average efficiency

Acknowledgments. This work has been supported by the national natural science foundation of china under grant no. 60503048 and 60672059 , the 863 program no. 2006AA01Z233.

\section{References}

1. Sarkar, V.: Partitionning and Scheduling Parallel Programs for Execution on Multiprocessors. MIT Press (1989)

2. Olivier, B., Vincent, B., Yves, R: The Iso-Level Scheduling Heuristic for Heterogeneous Processors, Proc. Of 10th Euromicro Workshop on Parallel, Distributed and Networkbased Processing (2002)

3. Topcuoglu, H., Hariri, S., Wu, M.: Performance-Effective and Low-Complexity Task Scheduling for Heterogeneous Computing. IEEE Trans. on Parallel and Distributed Systems, (2002) 260-274

4. Selvakumar, S. Murthy, C.S.R.: Scheduling Precedence Constrained Task Graphs with Non-Negligible Intertask Communication onto Multiprocessors, IEEE Trans. on Parallel and Distributed Systems, (1994) 328-336

5. Lai, G.L.: Scheduling Communication-Aware Tasks on Distributed Heterogeneous Computing Systems, Proceedings of $24^{\text {th }}$ International Conference on Distributed Computing Systems Workshops (2004)

6. Sinnen, O., Sousa, L.A.: Communication Contention in Task Scheduling, IEEE Trans. Parallel and Distributed Systems (2005) 503-515 